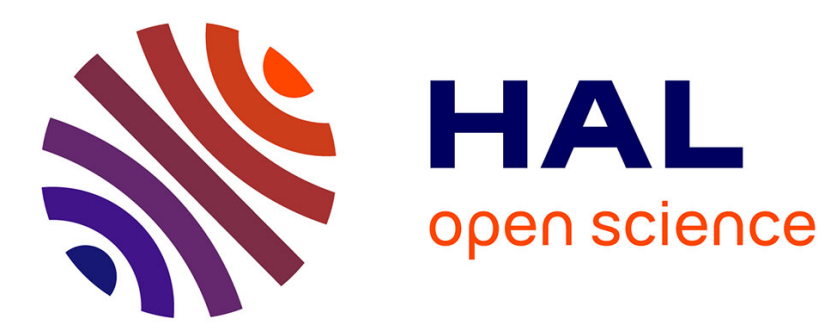

\title{
Selective amygdalohippocampectomy via trans-superior temporal gyrus keyhole approach
}

\author{
Bertrand Mathon, Stéphane Clemenceau
}

\section{To cite this version:}

Bertrand Mathon, Stéphane Clemenceau. Selective amygdalohippocampectomy via trans-superior temporal gyrus keyhole approach. Acta Neurochirurgica, 2016, 158 (4), pp.785-789. 10.1007/s00701016-2717-4 . hal-01290600

\section{HAL Id: hal-01290600 https://hal.sorbonne-universite.fr/hal-01290600}

Submitted on 18 Mar 2016

HAL is a multi-disciplinary open access archive for the deposit and dissemination of scientific research documents, whether they are published or not. The documents may come from teaching and research institutions in France or abroad, or from public or private research centers.
L'archive ouverte pluridisciplinaire HAL, est destinée au dépôt et à la diffusion de documents scientifiques de niveau recherche, publiés ou non, émanant des établissements d'enseignement et de recherche français ou étrangers, des laboratoires publics ou privés. 


\section{Selective amygdalohippocampectomy}

via trans-superior temporal gyrus keyhole approach

\section{Bertrand MATHON ${ }^{1,2}$, Stéphane CLEMENCEAU ${ }^{1}$.}

1 Department of Neurosurgery, La Pitié-Salpêtrière Hospital, AP-HP, Paris, France.

2 Sorbonne University, UPMC, Univ Paris 06, Paris, France.

\section{Corresponding author:}

Dr Bertrand MATHON

Department of Neurosurgery, Groupe Hospitalier Universitaire de La Pitié-Salpêtrière

47-83, Boulevard de l'Hôpital

75013 Paris, France.

Email: bertrand.mathon@,neurochirurgie.fr

\section{Conflict of interest}

All authors certify that they have NO affiliations or involvement with any organization or entity with any financial interest (such as honoraria; educational grants; participation in speaker's bureau; membership, employment, consultancies, stock ownership or other equity interest; and expert testimony of patent-licensing arrangements) or non- financial interest (such as personal or professional relationships, affiliations, knowledge or beliefs) in the subject matter or materials discussed in this manuscript. 


\begin{abstract}
Background. Hippocampal sclerosis is the most common cause of drug-resistant epilepsy amenable for surgical treatment and seizure control. The rationale of the selective amygdalohippocampectomy (SAH) is to spare cerebral tissue not included in the seizure generator.

Method. Describe the SAH through the trans-superior temporal gyrus keyhole approach.

Conclusion. SAH for temporal lobe epilepsy is performed when the data (semiology, neuroimaging, electroencephalography) point to the mesial temporal structures. Trans-superior temporal gyrus keyhole approach is a minimally invasive and safe technique that allows disconnection of the temporal stem and resection of temporomesial structures.
\end{abstract}

\title{
Keywords
}

Epilepsy surgery

Hippocampal sclerosis

Selective amygdalohippocampectomy

Transcortical approach

Mesial temporal lobe epilepsy 


\section{Relevant surgical anatomy}

The neural structures constituting the mesial temporal lobe are the parahippocampal gyrus (PHG), uncus, hippocampus, fimbria, dentate gyrus and amygdala [6, 10] (Fig. 1). With the temporal horn of the lateral ventricle opened, the hippocampus appears clearly visible. The hippocampus lies just lateral to the cerebral peduncle. Its head occupies the rostral extent of the temporal horn and curves mesially to form the uncus. Its body extends posteriorly into the tail that curves backward and upward to the trigone of the ventricle [2]. Inside the temporal horn the choroid plexus that partially covers the hippocampus and the choroid fissure are important surgical landmarks. Inferior choroidal point marks the beginning of the choroid fissure that lies between the fimbria and the thalamus. The hippocampal cisternal aspect faces the brainstem from which it is limited by the transverse fissure [2]. The crural cistern contains the P2 segment of the posterior cerebral artery and the oculomotor nerve. The hippocampal sulcus, containing the hippocampal arteries and veins, is perpendicular to the ambient cistern and separates the dentate gyrus superiorly from the parahippocampal gyrus inferiorly [8].

\section{Description of the technique}

Standard endotracheal general anesthesia is used. Patient is positioned supine with the shoulder elevated and the head turned. The patient's head is immobilized either in the Mayfield pin head holder, or with adhesive tape. The head is turned about $75^{\circ}$ contralaterally while being extended to make the zygomatic arch the highest point of the operative field. 
Scalp incision is a small curvilinear shape that starts at the superior border of the zygomatic arch just in front of the tragus, extends to the superior temporal line and anteriorly to the hairline (Fig. 2A). The temporalis muscle is incised via a curvilinear incision and retracted anteriorly.

Two burr holes are placed of each side of the sphenoid wing. A small temporal craniotomy is fashioned to expose the superior temporal gyrus (STG), the superior temporal sulcus and the Sylvian fissure. The sphenoid wing is flattened with a rongeur to allow adequate exposure to the anterior part of the STG (Fig. 2B). Hemostasis of the middle meningeal artery with cautery and sphenoid wing with bone wax is achieved.

The dura is opened in semicircular fashion and reflected over the temporalis muscle (Fig. 3A). A corticectomy measuring about $2 \mathrm{~cm}$ is made, as anterior as possible, along the upper border of the STG and just below the Sylvian fissure (Fig. 3B). Using an ultrasonic aspirator, a corridor is made along and directed by the arachnoid of the Sylvian fissure that points to the temporal horn (Fig. 3C). The ventricle is opened and retractors are positioned into the temporal horn to better visualize the choroid plexus and choroidal fissure marking the medial boundary of the hippocampal dissection (Fig. 3D). The hippocampus becomes visible.

The resection of the mesial structures is subpial using the ultrasonic dissector. The resection is started by entering the lateral ventricular sulcus at the junction of the hippocampus with the collateral eminence. A disconnection is made lateral to the hippocampus along the ventricular border lateral to the collateral eminence, in the anteroposterior axis, aiming to the level of the collateral sulcus (Fig. 3E). This allows the hippocampus to be retracted laterally to facilitate dissection of the fimbria 
attaching to the choroid fissure and disconnection mesially (Fig. 3F). The medial part of the parahippocampal gyrus is also separated of the arachnoid of the hippocampal sulcus. The combined hippocampus/PHG is separated from the hippocampal tail posteriorly by mediolateral transverse section. The specimen is freed from its arachnoidal cover by rolling it from mesial to lateral and separated from its vascular pedicle with a dissector (Fig. 4 A-B). The hippocampal formation should be removed en bloc to allow a finest histological examination [1] (Fig. 4 C, F). The typical posterior limit of the resection corresponds to the midbrain tectum (Fig. 4D). The anterior limit is the level of the rhinal sulcus that includes the uncus. The uncus and the amygdala are removed subpial. The mesial boundary of the amygdala resection is not precise. The roof of the ventricle can be used as a landmark to identify the extent of the amygdala resection. The amygdala is resected with ultrasonic dissector until the arachnoid membrane is encountered. Additional pieces of the hippocampal formation can be removed piecemeal, taking care to keep the pial surface intact.

Hemostasis is accomplished with Surgicel®, cottonoid packing and irrigation. The resection cavity is backfilled with irrigation (Fig. 4E). Dura is closed with running sutures in watertight fashion. Fibrin glue can be added on the dural closure to enhance watertightness. If epidural hemostasis needs to be achieved, dural tack-up sutures are placed along craniotomy edges. The bone flap is reaffixed with four non-absorbable sutures. The bone defect from removal of sphenoid wing can be eliminated with the bone powder. The wound is closed in standard fashion.

\section{Indications}

Selective amygdalohippocampectomy is indicated for patients presenting medically 
drug-resistant epilepsy localized to the temporomesial structures associated with hippocampal sclerosis.

Transcortical STG keyhole approach is a minimally invasive technique that allows disconnection of the temporal stem (unlike MTG approach) and resection of temporomesial structures (Fig. 5).

\section{Limitations}

Patients without clear evidence of mesial temporal lobe seizure foci, or involvement of lateral temporal neocortex and patients with cryptogenic temporal epilepsy are not ideal candidates for selective amygdalohippocampectomy. In such cases, anterolateral temporal lobectomy or tailored temporal cortectomy should be chosen as the appropriate technique.

The procedure we have described is strictly guided by anatomical landmarks, but image-guidance may help to localize these landmarks. This approach requires training, habit and a perfect knowledge of the temporal lobe anatomy. However, we recommend to use an image-guidance system for the first cases in order to better locate the landmarks of the corticectomy.

\section{How to avoid complications}

- Do not try to do the amygdalohippocampectomy without appropriate transventricular exposure. 
- Subpial dissection technique is a key to temporomesial resection. Take care to use the ultrasonic aspirator on low settings of suction and vibration to preserve the arachnoid protecting the critical underlying structures (cranial nerve III, posterior cerebral artery, anterior choroidal artery, midbrain peduncle).

- Take care not to extent the amygdala resection too superiorly into the globus pallidus.

- Be careful not to resect any tissue posterosuperior to the temporal horn to avoid damaging the fibers of Meyer's loop. Moreover, excessive posterolateral opening of the ventricle wall can result in damage to the optic radiations and leads to visual field defect.

- The hippocampal vessels typically can be separated from the hippocampus without coagulation. However, if coagulation and section of the hippocampal vessels in the hippocampal sulcus is necessary, take care to preserve the anterior choroidal artery and the posterior cerebral artery (P2 segment).

- Hemostasis of the resection cavity is accomplished with Surgicel ${ }^{\circledR}$ and cottonoid packing. Use of bipolar cautery in the arachnoid resection can be dangerous.

\section{Specific perioperative considerations}

Presurgical non-invasive evaluation comprised: complete neurological, neuropsychological and psychiatric examination, scalp video-EEG recording of habitual seizures, brain 3-Tesla MRI, and sometimes functional MRI, subtraction ictal single photon emission computed tomography (SPECT) co-registered to MRI and interictal positron emission tomography (PET) [5]. 
In postoperative period, patient is monitored at the intensive care unit and extubated when fully awake. Antiepileptic drugs must be resumed at full dose. A postoperative imaging (e.g. MRI) is performed to eliminate the occurrence of intracranial complication and evaluate the extent of temporomesial resection. Patient is carefully monitored for seizures.

\section{Specific information to give to the patient about surgery $\&$ potential risks}

Patients should be informed that the seizure-free rate after surgical resection for hippocampal sclerosis was about $70 \%[3,4]$. Mortality is very rare $(<1 \%)[9]$ and the rate of complication is low: definitive hemiparesis or third cranial nerve paralysis occurs in $1 \%$ of the cases [7]. Visual field defects occur frequently after surgery for hippocampal sclerosis and are usually contralateral superior quadrantanopia due to direct trauma to the optic radiations while accessing the mesial temporal structures.

\section{Key points}

- Knowledge of mesial temporal lobe anatomy is fundamental.

- Place the retractor to secure an unobstructed view of the intraventricular mesial temporal lobe structures.

- Visualize the choroid plexus and the choroid fissure that are most important surgical landmarks. 
- Subpial dissection technique is a key to all aspects of temporomesial epilepsy surgery.

- Be careful not to resect any tissue posterosuperior to the temporal horn to avoid damaging the fibers of Meyer's loop.

- Take care not to extent the amygdala resection too superiorly into the globus pallidus.

- Perform the posterior disconnection at the level of the midbrain tectum.

- The hippocampal vessels typically can be separated from the hippocampus without coagulation.

- Hemostasis of the resection cavity is accomplished with Surgicel ${ }^{\circledR}$ and cottonoid packing.

- In postoperative period, antiepileptic drugs must be resumed at full dose.

\section{Conflict of interest}

All authors certify that they have NO affiliations or involvement with any organization or entity with any financial interest (such as honoraria; educational grants; participation in speaker's bureau; membership, employment, consultancies, stock ownership or other equity interest; and expert testimony of patent-licensing arrangements) or non- financial interest (such as personal or professional relationships, affiliations, knowledge or beliefs) in the subject matter or materials discussed in this manuscript. 


\section{Acknowledgments}

The authors thank Dr. Dominique Hasboun and Dr. Anne Bertrand (Department of Neuroradiology, La Pitié-Salpêtrière Hospital, Paris, France) for anatomical views and imaging.

\section{References}

1. Blumcke I, Cross JH, Spreafico R (2013) The international consensus classification for hippocampal sclerosis: an important step towards accurate prognosis. Lancet Neurol 12:844-846

2. Destrieux C, Bourry D, Velut S (2013) Surgical anatomy of the hippocampus. Neurochirurgie 59:149-158

3. Dupont S, Tanguy ML, Clemenceau S, Adam C, Hazemann P, Baulac M (2006) Long-term prognosis and psychosocial outcomes after surgery for MTLE. Epilepsia 47:2115-2124

4. Mathon B, Bedos Ulvin L, Adam C, Baulac M, Dupont S, Navarro V, Cornu P, Clemenceau S (2015) Surgical treatment for mesial temporal lobe epilepsy associated with hippocampal sclerosis. Rev Neurol (Paris) 171:315-325

5. Mathon B, Bedos-Ulvin L, Baulac M, Dupont S, Navarro V, Carpentier A, Cornu P, Clemenceau S (2015) [Evolution of ideas and techniques, and future prospects in epilepsy surgery]. Rev Neurol (Paris) 171:141-156 
6. Sajko T, Skoro I, Rotim K (2013) How I do it - selective amygdalohippocampectomy via subtemporal approach. Acta Neurochir (Wien) $155: 2381-2387$

7. Tanriverdi T, Ajlan A, Poulin N, Olivier A (2009) Morbidity in epilepsy surgery: an experience based on 2449 epilepsy surgery procedures from a single institution. J Neurosurg 110:1111-1123

8. Wen HT, Rhoton AL, Jr., de Oliveira E, Cardoso AC, Tedeschi H, Baccanelli M, Marino R, Jr. (1999) Microsurgical anatomy of the temporal lobe: part 1: mesial temporal lobe anatomy and its vascular relationships as applied to amygdalohippocampectomy. Neurosurgery 45:549-591; discussion 591-542

9. Wiebe S, Blume WT, Girvin JP, Eliasziw M, Effectiveness, Efficiency of Surgery for Temporal Lobe Epilepsy Study G (2001) A randomized, controlled trial of surgery for temporal-lobe epilepsy. N Engl J Med 345:311-318

10. Wieser HG, Yasargil MG (1982) Selective amygdalohippocampectomy as a surgical treatment of mesiobasal limbic epilepsy. Surg Neurol 17:445-457

\section{Figures}

Fig. 1: Morphological and surgical anatomy of mesial temporal lobe. A. Axial slice of a right temporal lobe. 1, temporal pole; 2, hippocampal uncus; 3, amygdala; 4, hippocampus body (cornu Ammonis); 5, parahippocampal gyrus (T5); 6, lateral temporal neocortex. B. Frontal slice of a left mesial temporal lobe. 1, hippocampus body (cornu Ammonis); 2, parahippocampal gyrus (T5); 3, temporal horn of the lateral ventricle; 4, collateral sulcus. C. Anatomical pathway of a right trans-STG 
SAH. The amygdalohippocampal formation appears in yellow. The yellow bars delimit the trans-STG corridor.

Fig. 2: Scalp incision and craniotomy. A. Small curvilinear scalp incision for a left SAH. B. Keyhole craniotomy centered on the left STG (visualized on a 3D postoperative CT scan).

Fig. 3: Intraoperative view of left SAH via trans-STG keyhole approach (first part). A. The craniotomy is centered on the STG. The dura is opened in semicircular fashion and reflected over the temporalis muscle. B. A corticectomy is made along the upper border of the STG and just below the Sylvian fissure. C. A trans-STG corridor is made along and directed by the arachnoid of the Sylvian fissure that points to the temporal horn. D. The retractors are positioned into the temporal horn of the ventricle. The choroidal fissure marks the medial boundary of the hippocampal dissection. E. A disconnection is made lateral to the hippocampus along the ventricular border lateral to the collateral eminence. F. The hippocampus is retracted laterally to facilitate dissection of the fimbria attaching to the choroid fissure and disconnection mesially.

Fig. 4: Intraoperative view of left SAH via trans-STG keyhole approach (last part). A and B. The hippocampus is freed from its arachnoidal cover by rolling it from mesial to lateral and separated from its vascular pedicle with a dissector. C. The hippocampus is removed en bloc. D. The posterior limit of the resection corresponds to the midbrain tectum. E. Final view of the temporal neocortex after trans-STG SAH. F. Coronal section of the hippocampus allowing histological examination. 
Fig. 5: Postoperative brain MRI six months after left SAH via trans-STG keyhole approach. T2-weighted coronal views (A and B) and T1-weighted axial view (C) showing the trajectory through the STG and the cavity after the amygdalohippocampectomy.

\section{Video legend}

The video shows the major steps of a left selective amygdalohippocampectomy via trans-superior temporal gyrus keyhole approach. In our institution, the actual length of the whole procedure is 75 minutes on average. 
Fig. 1
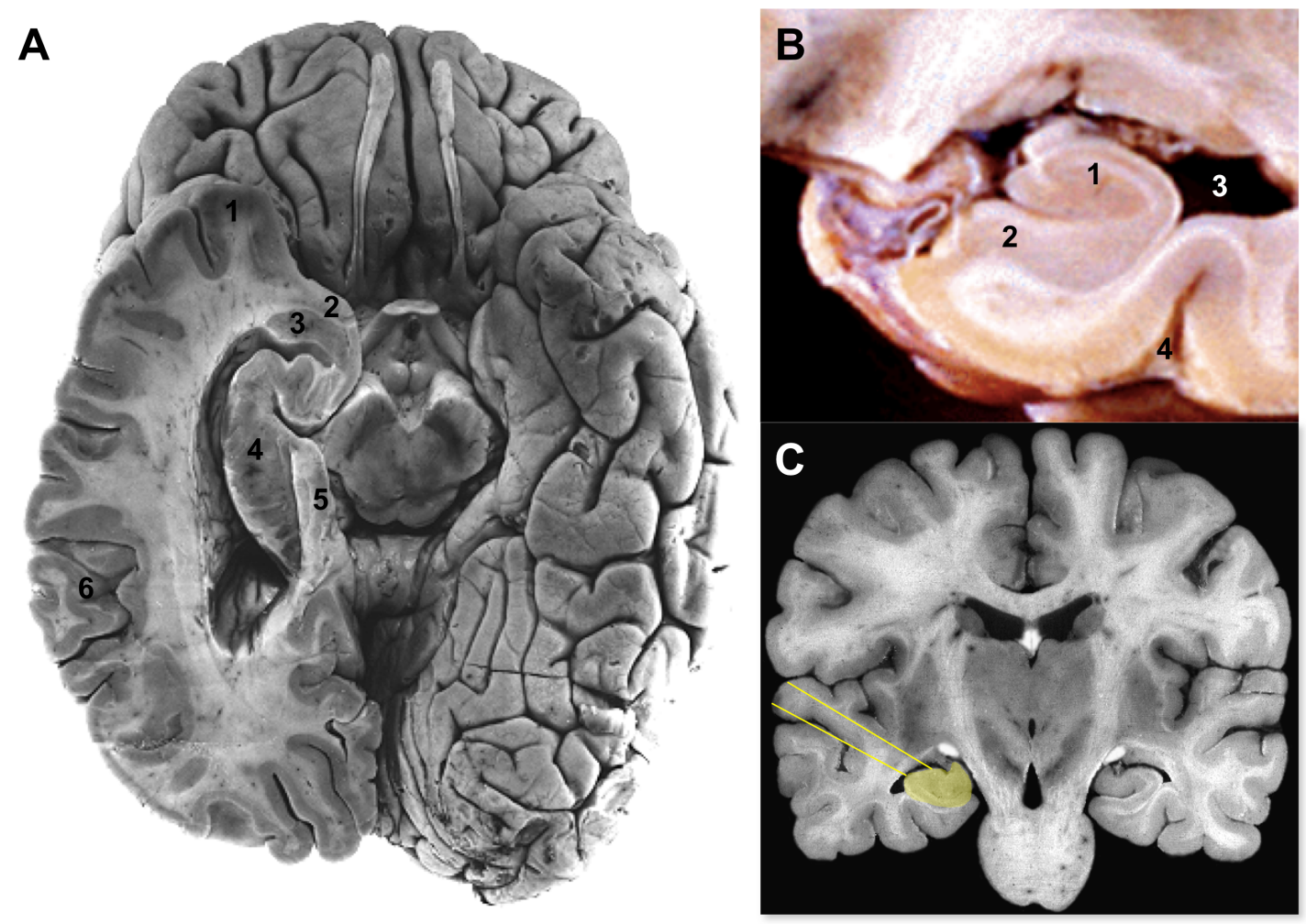

Fig. 2

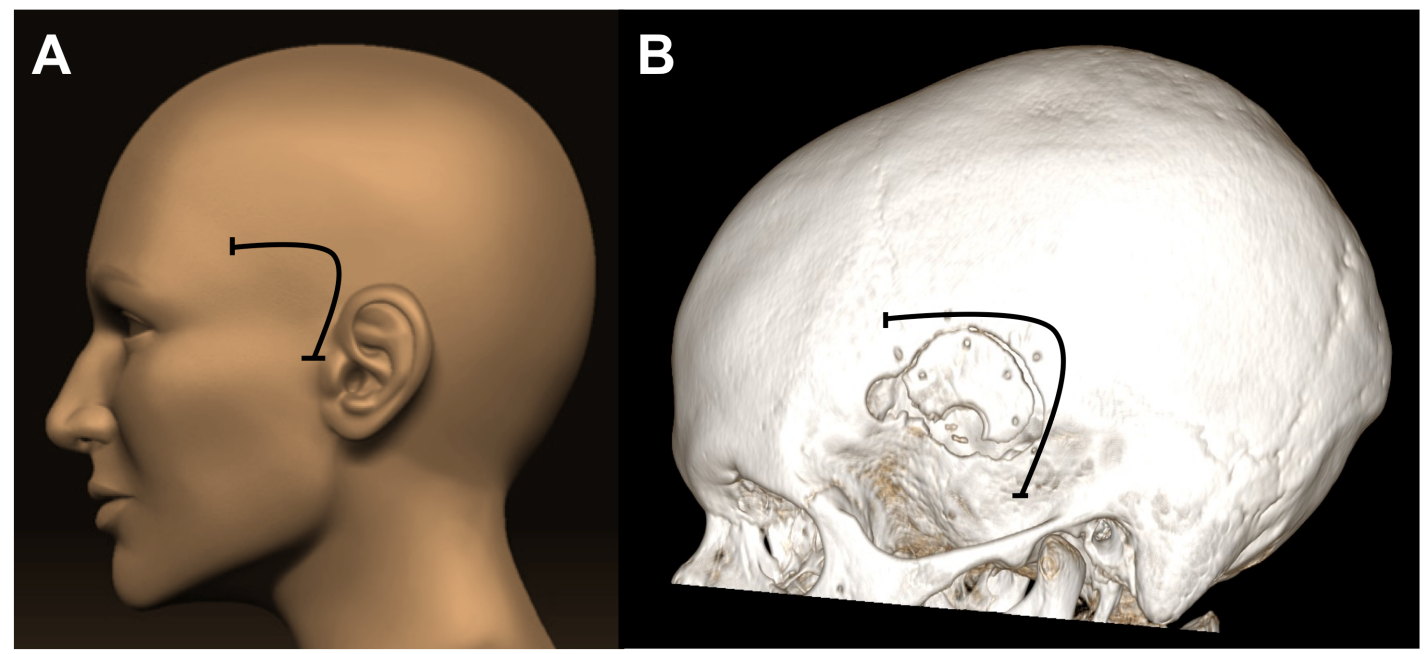


Fig. 3

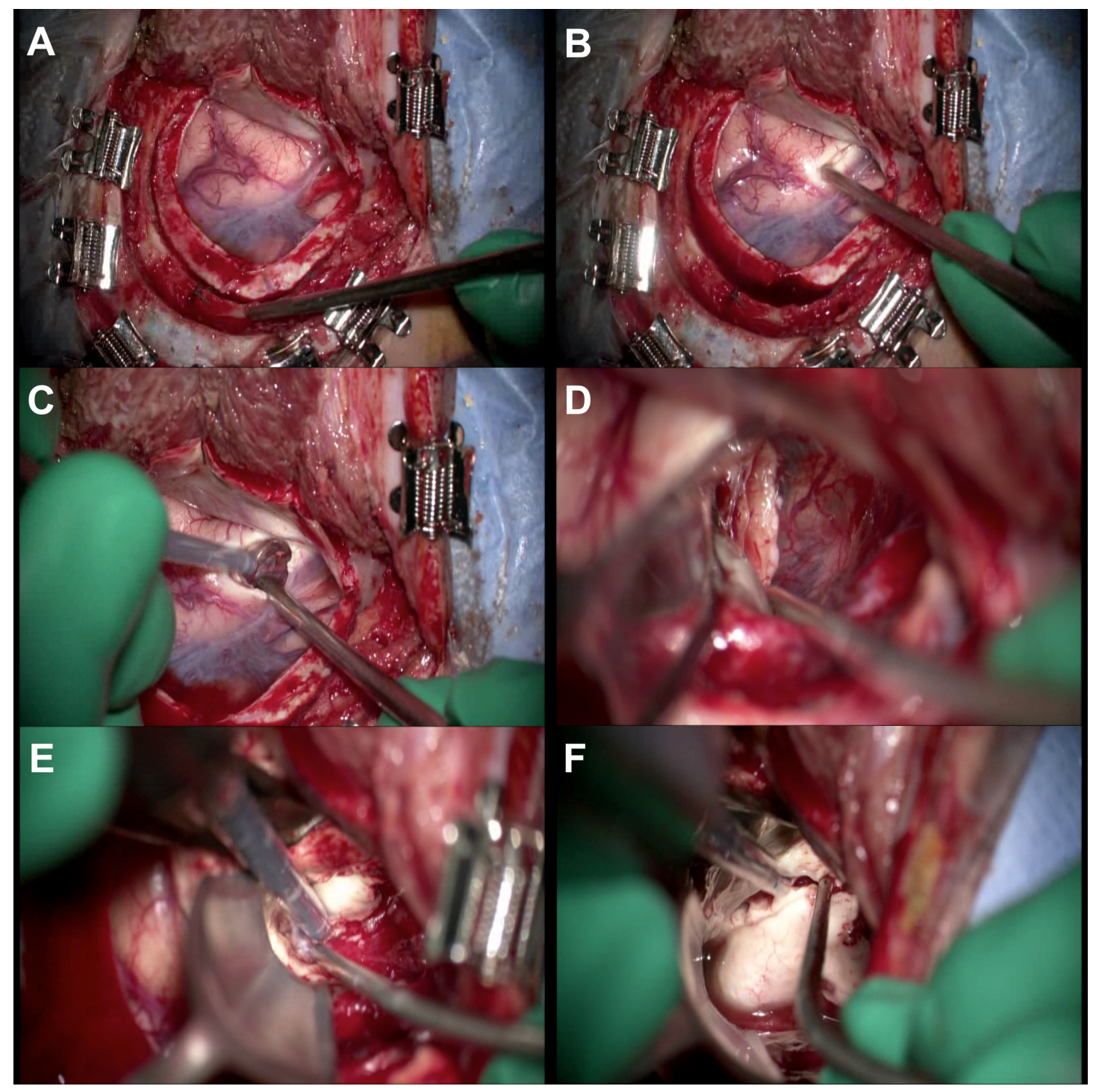


Fig. 4

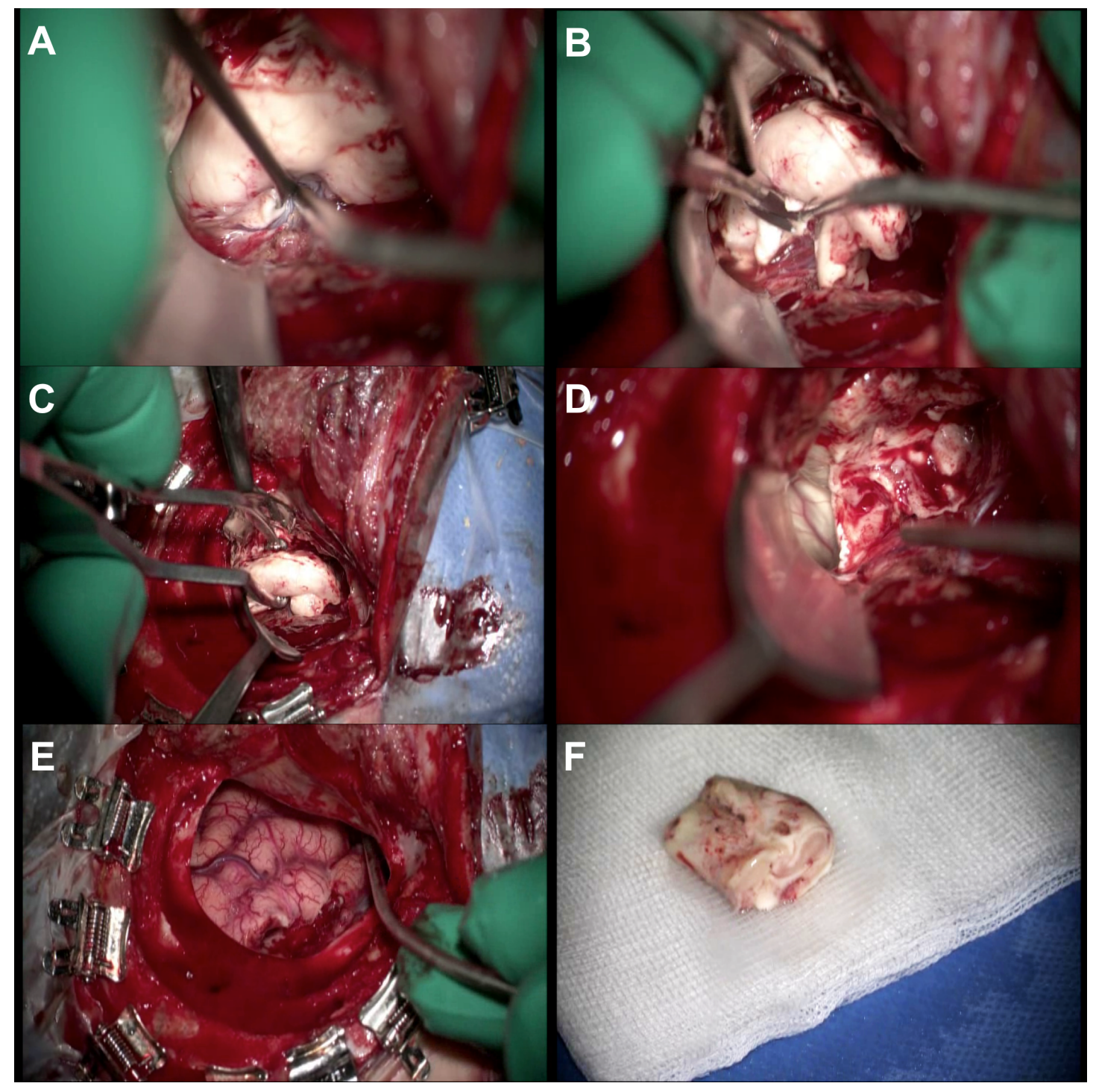

Fig. 5

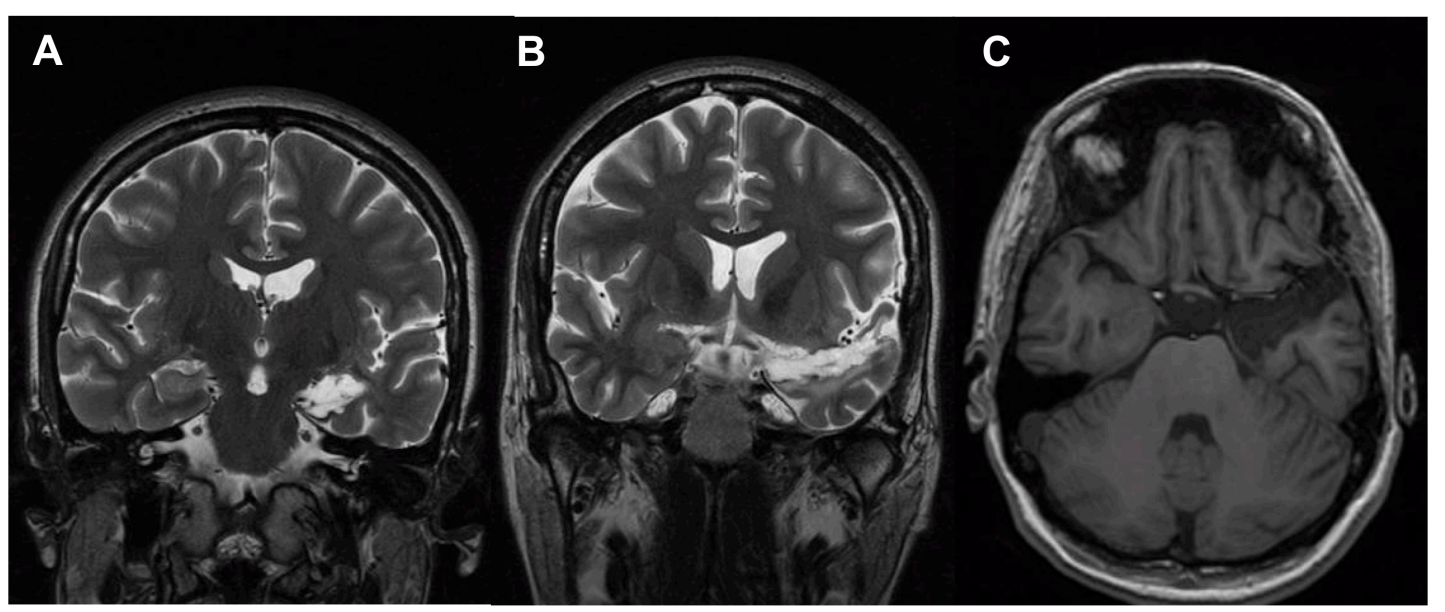

